\title{
Análise proteica, calórica e antropométrica de pacientes submetidos à fisioterapia convencional e cicloergômetro de membros inferiores em UTI: estudo piloto
}

\author{
Protein, caloric and anthropometric analysis of patients \\ submitted to conventional physiotherapy and cycle \\ ergometer of inferior members in ICU: a pilot study
}

\author{
Renata Cristina Dalves de Souza' (1) \\ Nayanne Paula de Andrade ${ }^{2}$ (1)
}

\author{
Eliane Maria de Carvalho 3 (i) \\ Fernanda Godoi Melo ${ }^{4}$
}

\footnotetext{
1,2,4Universidade Federal de Uberlândia (Uberlândia). Minas Gerais, Brasil.rcds.nutri@gmail.com, nayanne_paula@hotmail.com, ferngmelo@gmail.com ${ }^{3}$ Autora para correspondência. Universidade Federal de Uberlândia (Uberlândia). Minas Gerais, Brasil. elifisioufu@gmail.com
}

\begin{abstract}
RESUMO | INTRODUÇãO: A desnutrição proteico-energética é definida como resultado da deficiência calórica e/ou proteica, podendo causar alterações na composição corporal como a sarcopenia, no estado mental, na funcionalidade e consequentemente prejudicar o desfecho clínico. OBJETIVO: Avaliar oferta energética e proteica de pacientes críticos, submetidos à fisioterapia convencional associada ao cicloergômetro ativo (ou unicamente a fisioterapia convencional) e correlacionar com os dados antropométricos, e a escala MRC e Perme Escore. MATERIAL E MÉTODOS: estudo piloto com pacientes críticos internados na UTI, divididos entre grupo controle (GC) e experimental (GE). Todos foram avaliados pela escala MRC e PERME escore e submetidos a medidas antropométricas. Após 8 dias de intervenção fisioterapêutica convencional (GC), ou clicoergômetro ativo associado a abordagem convencional (GE), eles foram reavaliados. O aporte proteico e calórico de todos foram avaliados todos os dias. RESULTADOS: Houve redução significativa de medidas de circunferência de panturrilha no GC e perda não significativa de medidas no $\mathrm{GE}(\mathrm{p}=0,001)$. $O$ nível de adequação calórico e proteico foi de $73,9 \%$ e $69,5 \%$ respectivamente. Não houve diferença significativa entre os grupos. CONCLUSÃO: A mobilização com o cicloergômetro parece exercer efeito positivo na preservação da massa muscular em membros inferiores de pacientes críticos. No entanto, estudos que avaliam a medida de massa muscular em membros inferiores de modo mais controlado são necessários para comprovar essa hipótese.
\end{abstract}

PALAVRAS-CHAVE: Composição corporal. Desnutrição. Nutrição enteral. Terapia nutricional. Unidade de Terapia Intensiva.
ABSTRACT | INTRODUCTION: Protein-energy malnutrition is defined by caloric and/or protein deficiency, which can cause changes in body composition such as sarcopenia, mental status, functionality and consequently impair the clinical outcome. OBJECTIVE: To assess the energy and protein supply of critically ill patients undergoing conventional physical therapy associated with an active cycle ergometer (or only conventional physical therapy) and correlate with anthropometric data and the MRC scale and PERME score. MATERIALS AND METHODS: A pilot study with critically ill patients admitted to the ICU, divided into a control (CG) and experimental (EG) group. All were evaluated by the MRC scale and PERME score and submitted to anthropometric measurements. After eight days of conventional physical therapy intervention (CG) or active ergometer associated with the conventional approach (GE), they were reassessed. The protein and caloric intake of all were evaluated every day. RESULTS: There was a significant reduction in calf circumference measurements in the CG and a non-significant loss of measurements in the EG $(p=0.001)$. The caloric and protein adequacy level was $73.9 \%$ and $69.5 \%$, respectively. There was no significant difference between the groups. CONCLUSION: Mobilization with a cycle ergometer seems to positively affect the preservation of muscle mass in lower limbs in critically ill patients.

KEYWORDS: Body composition. Malnutrition. Enteral nutrition. Nutrition therapy. Intensive Care Units. 


\section{Introdução}

A desnutrição proteico-energética é definida como resultado da deficiência calórica e/ou proteica, podendo causar alterações na composição corporal como a sarcopenia, no estado mental, na funcionalidade e prejudicar o desfecho clínico. É causada por múltiplos fatores, como privação alimentar, doenças e/ou idade avançada. ${ }^{1}$ É uma condição comumente encontrada no ambiente hospitalar que leva ao aumento do risco de complicações cirúrgicas e infecciosas, desenvolvimento de lesões por pressão, mortalidade, tempo de internação e gastos hospitalares. ${ }^{2}$ Em unidades de terapia intensiva (UTI), a prevalência de subnutrição varia entre 43 e $88 \%$. 3

Em pacientes críticos ocorrem grandes perdas de massa muscular, e as causas são multifatoriais. Estão entre elas a contratilidade reduzida das fibras musculares, o desuso e degradação muscular, enquanto o anabolismo proteico é insuficiente para compensar essas perdas., ${ }^{4,5}$ Já foi demonstrado que esse estado hipermetabólico pode persistir por até dois anos após a alta hospitalar, prolongando o restabelecimento integral dos pacientes, destacando a dificuldade em recuperar massa magra e sua funcionalidade. ${ }^{6}$

No primeiro ano após internação, quase metade dos pacientes não retornaram ao trabalho e a mortalidade continua alta. Por isso, além das consequências intra-hospitalares citadas, o pós-alta também vem ganhando atenção especial. No mesmo ritmo que a mortalidade na UTI tem declinado, o número de pacientes que não retornam para a vida funcional pósUTI e que dependem de ambientes de reabilitação no pós-alta tem crescido. Esse "evento" tem sido chamado de post-intensive cares syndrome (síndrome do cuidado pós-intensivo). Sendo assim, os estudos que buscam medir a qualidade de vida após internação em UTI (principalmente no que diz respeito à funcionalidade física) tem conquistado espaço na literatura. $\frac{6}{}$ Para Wischmeyer et al., a nutrição e a atividade física ofertadas durante a internação são fatores essenciais nesse processo, pois dizem sobre a reserva metabólica do indivíduo. $\underline{6}$

É essencial que a equipe multidisciplinar desenvolva estratégias para minimizar as perdas da função muscular e capacidade funcional de pacientes internados em UTIs, visando incrementar a reabilitação nutricional e física permitindo que esses indivíduos sejam reintroduzidos na sociedade com independência funcional e qualidade de vida. Logo, o objetivo deste estudo foi avaliar a oferta energética e proteica de pacientes críticos. Estudos estes que estão submetidos à fisioterapia convencional associado ao cicloergômetro ativo de membros inferiores ou unicamente à fisioterapia convencional, correlacionando os dados antropométricos com a escala Medical Research Council (MRC) e o Perme Intensive Care Unit Mobility Score (PERME escore). Buscando confirmar o benefício e importância do aporte nutricional adequado e dos exercícios ativos para manutenção de massa muscular em pacientes críticos.

\section{Material e métodos}

Estudo piloto, com pacientes críticos internados na UTI de um hospital público universitário. A coleta dos dados foi de fevereiro a novembro de 2018. O estudo foi aprovado pelo comitê local de ética em pesquisa (CAAE: 82259317.9.0000.5152) e pelo Registro Brasileiro de Ensaios Clínicos (U1111-1215-1362).

Participaram do estudo pacientes que eram maiores de 18 anos (sem distinção de sexo e da causa de internação na UTI) que apresentaram um MRC de membros inferiores igual a 3, sendo aptos a realizar cicloergômetro ativo de membros inferiores. Dependentes de ventilação mecânica por mais de 48 horas. Pacientes que fossem capazes de seguir comandos simples e estivessem hemodinamicamente estáveis. Foram respeitados os critérios de estabilidade hemodinâmica e segurança da mobilização ativa propostos por Bradley e colaboradores. ${ }^{7}$ Foram excluídos aqueles pacientes que não fossem capazes de seguir aos comandos para realizar o cicloergômetro.

A escala MRC consiste na avaliação da força muscular através de seis movimentos testados bilateralmente. O grau de força muscular para cada movimento varia entre 0 (paralisia total) e 5 (força muscular normal). A pontuação total varia de 0 (tetraparesia completa) à 60 (força muscular normal). $\stackrel{8}{ }$ 
Todos foram avaliados pelo Perme Escore, que foi desenvolvido para avaliação do status de mobilidade de pacientes internados em UTI. O Perme Escore avalia através de quinze itens o status de mobilidade do paciente crítico. Dividido em sete categorias: estado mental, potenciais barreiras à mobilidade, força funcional, mobilidade no leito, transferências, endurance e distância percorrida. $\mathrm{O}$ escore varia de 0 a 32 pontos, onde uma baixa pontuação reflete menor mobilidade e maior necessidade de assistência, e o inverso se faz verdadeiro. ${ }^{-}$Ambas as escalas foram aplicadas por um fisioterapeuta.

O cálculo amostral foi realizado por meio do software G*Power 3.1.2.9. Para determinar o tamanho da amostra, utilizamos o cálculo para duas médias entre amostras dependentes. Os parâmetros utilizados foram: duas caudas, tamanho de efeito grande $(0,50)$; nível de significância padrão $(\alpha=0,05)$; poder do teste $(1-\beta=0,80)$, resultando em 34 pacientes.

Após elegíveis os pacientes foram randomizados em dois grupos, com envelopes lacrados, por pessoa não envolvida no estudo e classificados como Grupo Controle (GC) e Grupo Experimental (GE). Grupo controle (GC): receberam apenas a intervenção padrão do setor composta por exercícios ativo-assistidos ou ativos no leito de membros superiores e membros inferiores sem utilizar o cicloergômetro, com duração de 15 minutos, duas vezes ao dia e por oito dias consecutivos. Grupo experimental (GE): submetido à fisioterapia convencional, e à cicloergometria ativa de membros inferiores uma vez ao dia (Minibike para Exercícios Acte Sports $\left.{ }^{\circledR}\right)$, em decúbito dorsal e cabeceira elevada a 45 graus. O paciente pedalou ativamente o cicloergômetro durante 15 minutos, sem carga adicional. Foi respeitado o intervalo de 6 horas entre a intervenção convencional e o cicloergômetro.

No primeiro dia todos foram avaliados pela escala MRC e Perme Escore e submetidos às medidas antropométricas mais sensíveis a quantidade e funcionalidade muscular ${ }^{10}$, além de ser os membros mobilizados: circunferência de panturrilha (CP) e circunferência de braço (CB). No nono dia, após oito dias de intervenção, os participantes foram submetidos às mesmas avaliações do primeiro dia.
As medidas antropométricas foram aferidas nos membros superiores (CB) e inferiores (CP) que apresentavam melhores condições para aferição (mobilidade, sem curativos), sem investigar dominância do membro, mas no mesmo membro no primeiro e nono dia. Para obtenção da CP, a medida foi realizada com fita métrica inelástica. A perna dobrada em ângulo de 90 graus com o joelho e a medida é obtida na parte mais protuberante da panturrilha. Para a $C B$, primeiramente foi flexionado formando um ângulo de 90 graus, em seguida, localizamos o ponto médio entre as extremidades das proeminências do olecrano e da ulna, posteriormente, com o braço estendido, contornamos com fita métrica inelástica a altura do ponto médio, de forma que a fita ficasse aderida a pele, mas não a pressionasse. 11

Os dados referentes à necessidade e à oferta energética (em quilocalorias) e proteica (em gramas) foram coletados em prontuário diariamente. Assim como o valor do Simplified Acute Physiology Score (SAPS), que estima o risco de mortalidade do paciente..$^{12}$

Os dados foram analisados utilizando o programa SPSS versão 22.0. As variáveis quantitativas estão apresentadas em médias e desvio padrão e as qualitativas em frequências e porcentagens. Foi realizado Teste T para amostras independentes e assim avaliar diferenças entre grupos na escala MRC, Perme Escore, $\mathrm{CP}, \mathrm{CB}$, necessidade e oferta de quilocalorias e gramas de proteínas. As associações das variáveis qualitativas foram analisadas através do Teste Exato de Fisher, e as análises de correlação de Pearson entre dados antropométricos, oferta calórica e proteica, entre antropometria e valores da escala MRC e PERME escore finais. Nível de significância adotado: 95\%.

\section{Resultados}

Foram incluídos 33 pacientes. Entre esses, 10 evoluíram para óbito ou receberam alta da UTI antes de completar os 08 dias de intervenção. A amostra final foi de 23 pacientes, 11 no GE e 12 no GC. 


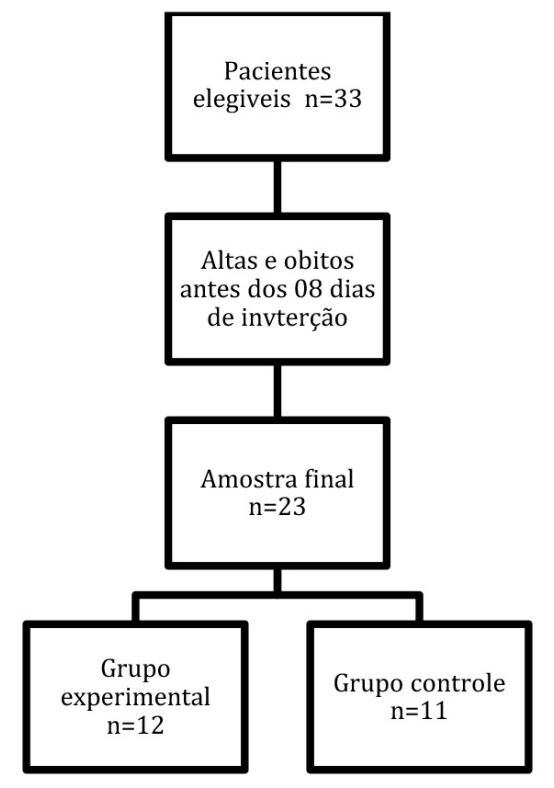

Na tabela 1 apresentamos a caracterização dos grupos com dados sociodemográficos, antropométricos, vias e tipos de alimentação onde maior parte dos participantes faziam uso de dieta enteral, via sonda nasoenteral. Não houve diferença significativa entre os grupos analisados. Apesar da maioria dos pacientes terem recebido quantidades médias de energia e proteínas próximas de suas necessidades na Tabela 2, o déficit energético e proteico

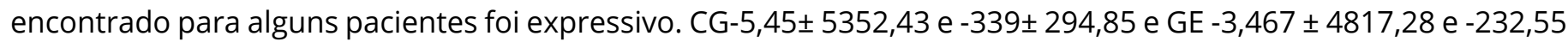
$\pm 290,20$, fram esses os valores para energia e proteína respectivamente.

Na Tabela 3 são apresentadas as porcentagens de adequação energética e proteica médias recebidas em relação às necessidades dos pacientes por grupo, sem significância estatística.

Na tabela 4 apresentamos as variáveis antropométricas analisadas antes e após intervenção fisioterapêutica. Quando analisadas a circunferência da panturrilha inicial (CPI) e no 90 dia a final (CPF), observamos redução significativa $(p=0,001)$. O GC não houve diferença no $G E(p=0,053)$. Comparando a medida da circunferência de braço inicial (CBI) com o 9o dia a final (CBF), observamos diminuição significativa nos dois grupos ( $G C p=0,038$ e GE $p=0,041)$.

Ao analisar a correlação do débito energético e proteico (diferença entre necessidade e oferta) e a redução de medidas antropométricas em ambos os grupos, não houve correlações significativas. Por fim, realizamos análises de correlação entre o débito energético e proteico e os valores finais da escala MRC e Perme Escore, observamos correlação significativa, moderada e inversa entre o débito energético e o Perme Escore final no GC, demonstrando que quanto mais calorias o paciente não recebesse, maior era sua pontuação no Perme Escore. As demais correlações não apresentaram significância, como pode ser visto na Tabela 5. 
Figura 2. Caracterização clínica dos pacientes críticos, $N=23$

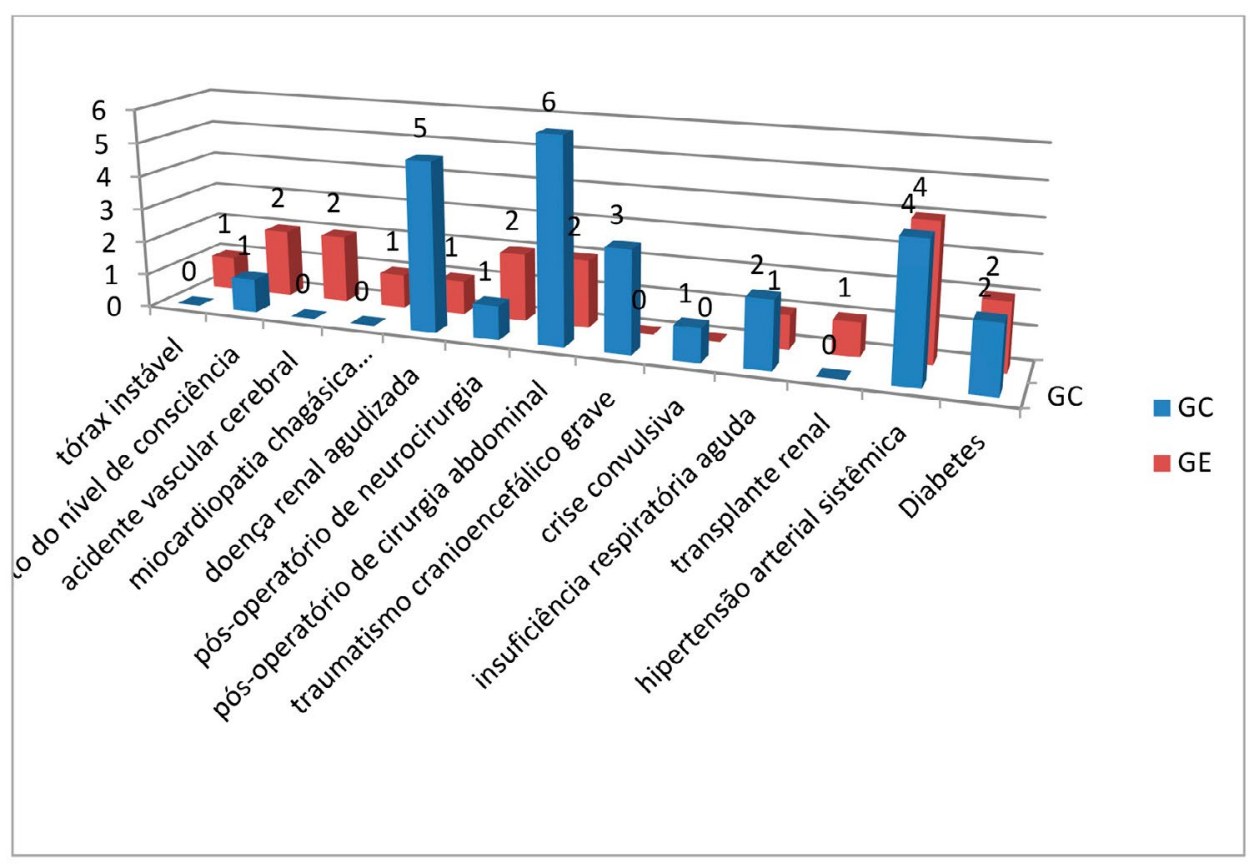

$\mathrm{N}=$ população do estudo

GC: grupo controle

GE: grupo experimental

Tabela 1. Caracterização dos pacientes críticos e vias de alimentação, $N=23$

\begin{tabular}{|c|c|c|c|}
\hline & $G C(n=12)$ & $G E(n=11)$ & $\mathbf{P}$ \\
\hline Homens/ Mulheres & $8 / 4$ & $6 / 5$ & \\
\hline Idade (anos) & $55,2 \pm 20,6$ & $49,5 \pm 17,1$ & 0,491 \\
\hline $\begin{array}{l}\text { Tempo de internação ao entrar na pesquisa } \\
\text { (dias) }\end{array}$ & $15,75 \pm 13,2$ & $11,91 \pm 6,3$ & 0,402 \\
\hline Tempo de internação total (dias) & $25,4 \pm 13,9$ & $24,9 \pm 10,3$ & 0,922 \\
\hline SAPS points & $66,4 \pm 17,5$ & $63,0 \pm 20,4$ & 0,671 \\
\hline Altura média (metros) & $1,71 \pm 0,63$ & $1,66 \pm 0,09$ & 0,318 \\
\hline Peso médio (quilos) & $71,7 \pm 5,73$ & $65,8 \pm 8,12$ & 0,214 \\
\hline $\mathrm{IMC}\left(\mathrm{kg} / \mathrm{m}^{2}\right)$ & $24,4 \pm 1,18$ & $23,8 \pm 1,67$ & 0,419 \\
\hline \multicolumn{4}{|l|}{ Via de alimentação* } \\
\hline Dieta SNE(n/\%) & $5(41,6)$ & $7(63,6)$ & \\
\hline Dieta SNE e VO(n/\%) & $3(25)$ & $3(27,2)$ & \\
\hline$N P(n / \%)$ & $2(16,6)$ & $1(9,0)$ & \\
\hline Dieta SNE e NP(n/\%) & $2(16,6)$ & $0(0)$ & 0,230 \\
\hline \multicolumn{4}{|l|}{ Desfecho clínico** } \\
\hline Altas(n/\%) & $8(74,9)$ & $8(64,4)$ & \\
\hline Óbitos(n/\%) & $4(36,6)$ & $3(25,1)$ & 0,554 \\
\hline
\end{tabular}

$\mathrm{N}=$ população do estudo

Teste de Fisher *

Teste $t$ Student ${ }^{\star *}$

SAPS: SimplifiedAcutePhysiology Score, SNE: sonda nasoentérica, VO: via oral, NP: Nutrição parenteral 
Tabela 2. Apresentação dos valores em quilocalorias e gramas que cada paciente crítico deixou de receber nos nove dias de acompanhamento. $\mathrm{N}=23$

\begin{tabular}{|c|c|c|c|}
\hline \multicolumn{2}{|c|}{ Energia(kcal) } & \multicolumn{2}{|r|}{ Proteínas(g) } \\
\hline Difere & $\begin{array}{l}\text { nça entre necessidade e } \\
\text { oferta }\end{array}$ & Paciente & $\begin{array}{c}\text { Diferença entre necessidade e } \\
\text { oferta }\end{array}$ \\
\hline \multicolumn{4}{|c|}{ GC } \\
\hline GC11 & -17.547 & GC11 & -911 \\
\hline GC01 & -11.075 & GC01 & -767 \\
\hline GC18 & -10.689 & GC18 & -588 \\
\hline GC13 & -8.574 & GC13 & -480 \\
\hline GC12 & -4.515 & GC12 & -411 \\
\hline GC06 & -3.900 & GC06 & -273 \\
\hline GC02 & -2.460 & GC08 & -230 \\
\hline GC08 & -2.192 & GCO2 & -131 \\
\hline GC10 & -2.043 & GC10 & -118 \\
\hline GC09 & -1.653 & GCO9 & -101 \\
\hline GC17 & -825 & GC17 & -58 \\
\hline GC07 & 0 & GCO7 & 0 \\
\hline Média e desvio padrão & $-5,45 \pm 5352,43$ & & $-339 \pm 294,85$ \\
\hline \multicolumn{4}{|c|}{ GE } \\
\hline GE05 & -15.750 & GE05 & -945 \\
\hline GE15 & -9.114 & GE15 & -569 \\
\hline GE10 & -4.256 & GEO9 & -293 \\
\hline GE01 & -2.133 & GE10 & -222 \\
\hline GE13 & -1.968 & GE01 & -156 \\
\hline GE11 & -1.964 & GE04 & -146 \\
\hline GE09 & -1.224 & GE11 & -112 \\
\hline GEO2 & -864 & GE13 & -84 \\
\hline GE07 & -700 & GEO2 & -78 \\
\hline GE04 & -164 & GE12 & -56 \\
\hline GE12 & 0 & GE07 & 103 \\
\hline \multicolumn{4}{|l|}{ Média e desvio } \\
\hline padrão & $-3,467 \pm 4817,28$ & & $-232,55 \pm 290,20$ \\
\hline
\end{tabular}

GC: grupo controle, GE: grupo experimental

Tabela 3. Porcentagem de adequação energética e proteica em relação às necessidadesdo paciente crítico. $\mathrm{N}=23$

\begin{tabular}{lllllll}
\hline & GC & & & & \\
& & GE & & Geral \\
& Energia(n/\%) & Proteína(n/\%) & Energia(n/\%) & Proteína(n/\%) & Energia(n/\%) & Proteína(n/\%) \\
$\mathbf{0}$ a $\mathbf{3 2 , 9 \%}$ & $2(16,6)$ & $3(25,0)$ & $1(9,0)$ & $2(18,1)$ & $3(13,0)$ & $5(21.7)$ \\
$\mathbf{3 3}$ a $\mathbf{6 5 , 9 \%}$ & $2(16,6)$ & $2(16,6)$ & $1(9,0)$ & $0(0)$ & $3(13,0)$ & $2(8,6)$ \\
$\mathbf{6 6}$ a $\mathbf{1 0 0 \%}$ & $8(66,6)$ & $7(58,3)$ & $9(81,8)$ & $9(81,8)$ & $17(73,9)$ & $16(69,5)$ \\
\end{tabular}

Tabela 4. Associação entre as variáveis antropométricasem paciente crítico, $\mathrm{N}=23$

\begin{tabular}{lccccccc}
\hline & \multicolumn{2}{c}{ Circunferência de Panturrilha $(\mathbf{c m})$} & \multicolumn{3}{c}{ Circunferência de Braço (cm) } \\
\cline { 2 - 8 } & \multicolumn{2}{c}{ Inicial } & Final & $\mathbf{p}^{\mathbf{a}}$ & Inicial & Final & $\mathbf{p}^{\text {a }}$ \\
\hline GC & $33,3 \pm 3,8$ & $31,1 \pm 3,4$ & 0,001 & $29,6 \pm 3,9$ & $27,9 \pm 3,7$ & 0,038 \\
GE & $33,0 \pm 3,3$ & $31,8 \pm 3,6$ & 0,053 & $30,0 \pm 3,6$ & $29,0 \pm 3,7$ & 0,041 \\
& 0,873 & 0,643 & & 0,836 & 0,474 & \\
\hline
\end{tabular}

Teste T Student

${ }^{a} p$ valor entre a medida inicial e final

${ }^{b} p$ valor entre GC e GE 
Tabela 5.Correlação do débito energético e proteico e a redução de medidas antropométricas e entre o débito energético e proteico e os valores finais da escala MRC e PERME escore. $\mathrm{N}=23$

\begin{tabular}{lrrrr}
\hline & GC & & GE & \\
\cline { 2 - 5 } & r & p & $r$ & $p$ \\
\hline Kcal vs CP & 0,2 & 0,52 & 0,45 & 0,15 \\
Kcal VS CB & 0,36 & 0,23 & 0,49 & 0,11 \\
Proteína vs CP & 0,24 & 0,43 & 0,33 & 0,3 \\
Proteína vs CB & 0,37 & 0,22 & 0,36 & 0,26 \\
Kcal vs PERME final & 0,59 & 0,03 & 0,31 & 0,35 \\
Kcal vs MRC final & 0,29 & 0,35 & $-0,01$ & 0,96 \\
$\begin{array}{l}\text { Proteína vs PERME } \\
\text { final }\end{array}$ & 0,52 & 0,08 & 0,43 & 0,17 \\
$\begin{array}{l}\text { Proteína vs MRC final } \\
\text { Fonte: Elaboração própria. }\end{array}$ & 0,2 & 0,52 & 0,05 & 0,87 \\
$\begin{array}{l}\text { Teste Sudent T } \\
\text { Kcal = quilocalorias } \\
\text { CP= circunferência de panturrilha } \\
\text { CB = circunferência de braço }\end{array}$ & & & & \\
\end{tabular}

\section{Discussão}

O objetivo deste estudo foi confirmar o benefício e importância do aporte nutricional adequado e dos exercícios ativos para manutenção de massa muscular em pacientes críticos.

Apesar da maioria dos pacientes estarem próximos das metas proteicas e calóricas, o nível de adequação ainda é baixo. Sendo $73,9 \%$ e $69,5 \%$ de adequação calórica e proteica, respectivamente, ficando abaixo de $80 \%$, conforme demonstrou Ribeiro. ${ }^{13}$ Resultados semelhantes foram encontrados por Menezes et al., onde os níveis de adequação calórica e proteica foram de 77,2\% e 73,8\%.14 Enquanto Nunes et al. obtiveram adequação calórica de 64,6\% e proteica de $66,7 \% .15$ As pesquisas apontam uma das justificativas para estes resultados se devem a quantidade de pausas feitas para procedimentos $(30,0 \%)$ e falhas na administração $(42,9 \%)$. ${ }^{16}$ Neste estudo, não foram analisados estes dados, caracterizando um viés da pesquisa.

O elevado déficit de calorias e proteínas é um fator preocupante, especialmente pela gravidade dos pacientes, que possuem um catabolismo proteico acentuado e aumento do gasto energético de repouso ${ }^{17}$, embora a oferta de nutrientes não possa reverter a proteólise, a gliconeogênese e a lipólise associadas ao estresse, podem reduzir as consequências do catabolismo exacerbado, com melhores resultados quando associados a mobilização precoce efetiva. $\frac{18}{}$

Em relação aos dados antropométricos, identificamos diminuições significativas de medidas de CP e CB no GC, e diminuição não significativa da CP no GE. Demonstrando assim os efeitos positivos para manutenção de massa muscular quando utilizado o cicloergômetro. Contudo, não se pode afirmar que apenas a mobilização seja suficiente para manutenção das medidas, uma vez que está bem instituída a importância da nutrição na manutenção das fibras musculares. 6 Estudos que avaliam de uma forma mais acurada as características da massa musculoesquelética são necessárias para confirmar essa hipótese.

Não houveram correlações significativas entre as medidas antropométricas e o déficit calórico ou proteico, ou entre déficit calórico ou proteico e as escalas. A correlação encontrada foi entre o déficit de quilocalorias e o Perme Escore final no GC, o que pode sugerir o inverso do esperado. A ausência de correlações entre as variáveis analisadas pode ter sido gerada devido ao n não ter sido atingido, e por isso a caracterização sociodemográfica e clínica não ter sido homogênea, pode se considerar uma das limitações do estudo. 
Estudos clínicos com uma amostra mais robusta, maior tempo de acompanhamento e analise de massa muscular mais fidedigna como a ultrassonografia, ou tomografia, por exemplo, seriam de grande importância para comprovar o que este estudo piloto sugere.

Como limitações do estudo, encontra-se o fato de que o número de pacientes não alcançou o tamanho amostral, possivelmente pelo perfil dos pacientes onde poucos possuíam nível de consciência para inclusão, além da escassez de estudos similares para discussão.

\section{Conclusão}

A mobilização com o cicloergômetro parece exercer efeito positivo na preservação da massa muscular em membros inferiores de pacientes críticos. No entanto, estudos que avaliem a medida de massa muscular de modo mais controlados em membros inferiores são necessários para comprovar essa hipótese.

\section{Agradecimentos}

Artigo oriundo do trabalho de conclusão de residência pelo Programa de Residência em Área Profissional da Saúde, Faculdade de Medicina, Universidade Federal de Uberlândia, baseado na dissertação "Análise da Mobilidade de pacientes críticos submetidos a Intervenção Fisioterapêutica em relação ao Perme Escore".

\section{Contribuições das autoras}

Souza RCD foi responsável pela coleta de dados e construção do manuscrito. Andrade NP foi responsável pela coleta de dados e revisão do manuscrito. Carvalho EM e Melo FG orientaram a pesquisa e revisaram a versão final do texto.

\section{Conflitos de interesses}

Nenhum conflito financeiro, legal ou político envolvendo terceiros (governo, empresas e fundações privadas, etc.) foi declarado para nenhum aspecto do trabalho submetido (incluindo, mas não se limitando a subvenções e financiamentos, participação em conselho consultivo, desenho de estudo, preparação de manuscrito, análise estatística, etc.).

\section{Referências}

1. Cederholm T, Barazzoni R, Austin P, Ballmer P, Biolo G, Bischoff SC, et al. ESPEN guidelines on definitions and terminology of clinical nutrition. Clin Nutr. 2017;36(1):49-64. https://doi. org/10.1016/j.clnu.2016.09.004

2. Santos CA, Firmino HH, Esmeraldo MLF, Alfenas RCG, Rosa $\mathrm{COB}$, Ribeiro AQ, et al. Perfil nutricional e fatores associados à desnutrição e ao óbito em pacientes com indicação de terapia nutricional. Braspen J [Internet]. 2017;32(1):30-5. Disponível em: http://www.posnutricao.ufv.br/eng/wp-content/uploads/2018/03/ Perfil-nutricional-e-fatores-associados-\%C3\%A0.pdf

3. Santana MMA, Vieira LL, Dias DAM, Braga CC, Costa RM. Inadequação calórica e proteica e fatores associados em pacientes graves. Rev Nutri. 2016;29(5):645-54. https://doi. org/10.1590/1678-98652016000500003

4. Mesquita TMJC, Gardenghi G. Imobilismo e fraqueza muscular adquirida na unidade de terapia. Rev Bras Saúde Func [Internet]. 2016;3(1):47-58. Disponível em: https://seer-adventista.com.br/ ojs3/index.php/RBSF/article/view/717

5. Calvo-Ayala E, Khan BA, Farber MO, Ely EW, Boustani MA. Interventions to improve the physical function of ICU survivors: a systematic review. Chest. 2013;144(5):1469-80. https://doi. org/10.1378/chest.13-0779

6. Wischmeyer PE, Puthucheary Z, San Millàn IS, Butz D, Grocott MPW. Muscle Mass and Physical Recovery in ICU: Innovations for Targeting of Nutrition and Exercise. Curr Opin Crit Care. 2017;23(4):269-78. Citado em: PMID: 28661414

7. Hodgson CL, Stiller K, Needham DL, Tipping CL, Harrold M, Baldwin CL, et al. Expert consensus and recommendations on safety criteria for active mobilization of mechanically ventilated critically ill adults. Crit Care. 2014;18(6):658. https://doi. org/10.1186/s13054-014-0658-y

8. Walton J, Gilliant RW, Hutchinson M, McArdle MJF, O'Brien $M D$, Thomas PK, et al. Aids to the examination of the peripheral nervous system. London, UK: Bailliere Tindall; 1986.

9. Perme C, Nawa RK, Winkelman C, Masud F. A tool to assess mobility status in critically ill patients: the Perme Intensive Care Unit Mobility Score. Methodist Debakey Cardiovasc J. 2014;10(1):41-9. https://doi.org/10.14797/mdcj-10-1-41

10. Peixoto LG, Barbosa CD, Nahas, PC, Rossato LT, Oliveira EP. A circunferência da panturrilha está associada com a massa muscular de indivíduos hospitalizados. Rev Bras Nutri Clin [Internet]. 2016;31(2):167-71. Disponível em: http://www. braspen.com.br/home/wp-content/uploads/2016/11/14-Acircunfer\%C3\%AAncia-da-panturrilha.pdf 
11. Cuppari L. Guia de nutrição: nutrição clínica no adulto. $3^{\mathrm{a}}$ ed. São Paulo: Manole; 2002.

12.Leão FGA, Marques IDB, Mello PMVC. Validação do índice prognóstico SAPS 3 em pacientes internados na UTI de um hospital terciário de Teresina (PI). J. Ciênc. Saúde Hosp. Univ. Univ. Fed. Piauí. 2018;1(3):9-19. https://doi.org/10.26694/25950290.2018139-197207

13. Ribeiro PC. Nutrição. São Paulo: Atheneu; 2015.

14. Menezes NNB, Silva JT, Brito LC, Gois FN, Oliveira CC. Adequação entre a terapia nutricional enteral prescrita e a dieta administrada em pacientes críticos. Nutr Clín Diet Hosp. 2018;38(4):57-64. https://doi.org/10.12873/384nara

15. Nunes AP, Zanchim MC, Kümpel DA, Rodrigues TP, Zanin J. Adequação calórico-proteica da terapia nutricional enteral em pacientes críticos de um hospital de alta complexidade do Rio Grande do Sul. Braspen J [Internet]. 2018;33(2):116-21. Disponível em: http://arquivos.braspen.org/journal/abr-mai-jun-2018/02-AOAdequacao-calorico-proteica.pdf

16. Silva RQ, Cardoso LG. Causas de Interrupção da Nutrição Enteral em Unidades de Terapia Intensiva [Monografia] [Internet]. Vitória da Conquista: Universidade Federal da Bahia; 2019. Disponível em: https://repositorio.ufba.br/ri/handle/ri/28969

17. Pereira TG, Fink JS, Tosatti JAG, Silva FM. Subjective Global Assessment Can Be Performed in Critically III Surgical Patients as a Predictor of Poor Clinical Outcomes. Nutr Clin Pract. 2019;34(1):131-36. https://doi.org/10.1002/ncp.10178

18. Hodgson CL, Tipping CJ. Physiotherapy management of intensive care unit-acquired weakness. J Physiother. 2017;63(1):410. https://doi.org/10.1016/j.jphys.2016.10.011 